\title{
Influence of Mg and In on defect formation in GaN; bulk and MOCVD grown samples
}

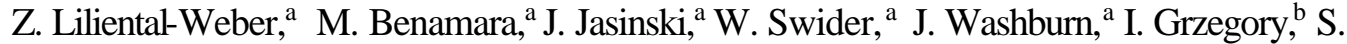 \\ Porowski, ${ }^{b}$ J. Bak-Misiuk, J. Domagala, ${ }^{c}$ S. Bedair, ${ }^{\mathrm{d}}$ C.J. Eiting ${ }^{\mathrm{e}}$, and R.D. Dupuis ${ }^{\mathrm{e}}$ \\ ${ }^{a}$ Lawrence Berkeley National Laboratory, Berkeley, CA 94720; USA, m/s 62/203 \\ ${ }^{b}$ High Pressure Research Center "Unipress," Polish Academy of Sciences, Warsaw, Poland, \\ ${ }^{c}$ Institut of Physics Polish Academy of Sciences, Warsaw, Poland; ${ }^{d}$ Materials Science and \\ Engineering, North Carolina State University, NC 27695; ${ }^{e}$ The University of Texas at Austin, \\ Microelectronics Research Center, PRC/MER 1.606D-R9900, Austin TX 78712-1100 USA
}

Abstract-Transmission electron microscopy studies were applied to study GaN crystals doped with Mg. Both: bulk GaN:Mg crystals grown by a high pressure and high temperature process and those grown by metal-organic chemical-vapor deposition (MOCVD) have been studied. Structural dependence on growth polarity was observed in the bulk crystals. Spontaneous ordering (formation of polytypoids) was observed for growth in the $\mathbf{N}$ to $\mathrm{Ga}$ polar direction ( $\mathrm{N}$ polarity). On the opposite site of the crystal (growth in the $\mathrm{Ga}$ to $\mathrm{N}$ polar direction) Mg-rich pyramidal defects with base on the basal planes and with walls inclined about $45^{\circ}$ to these planes, empty inside (pinholes) were observed. A high concentration of these pyramidal defects was also observed in the MOCVD grown crystals. For samples grown with Mg delta doping planar defects were also observed especially at the early stages of growth followed by formation of pyramidal defects.

TEM and $x$-ray studies of $\operatorname{In}_{x} \mathrm{Ga}_{1-\mathrm{x}} \mathrm{N}$ crystals for the range of $28-45 \%$ nominal In concentration shows formation of two sub-layers: strained and relaxed, with a much lower In concentration in the strained layer. Layers with the highest In concentration were fully relaxed.

\section{A. Introduction}

Possibility of doping for formation of $\mathrm{p}-\mathrm{n}$ junctions is important for any material in order to apply it as a device material. GaN can easily be grown with n-conductivity but obtaining p-doping is rather difficult. $\mathrm{Mg}$ is most commonly used as the p-dopant in $\mathrm{GaN}$, however, higher hole concentrations can only be obtained after thermal annealing [1,2] in order to dissociate $\mathrm{Mg}-\mathrm{H}$ complexes. Material made using this process has been used to fabricate light emitting diodes (LEDs) [3] and lasers [4]. Despite this success, many aspects of Mg-doping in GaN are still not fully understood. Transmission electron microscopy studies (TEM) show formation of different types of Mg-rich defects. The type of defects formed depends strongly on the growth polarity. This can be easily observed in bulk GaN:Mg platelete crystals where both growth polarities can be observed in one plate. For growth with N-polarity a superlattice-like array of planar defects (polytypoids) leading to superlattice reflections in the diffraction pattern can be observed [5-7]. Similar defects are also formed in layers grown by metałorganic-chemical -vapor-deposition (MOCVD) with $\mathrm{Mg}$ de lta doping [6-7]. Growth with $\mathrm{Ga}$ polarity leads to formation of pyramidal and rectangular defect, that are empty inside. These pyramidal defects are typical for all MOCVD-grown crystals. A high concentration can be observed in all crystals for a wide range of $\mathrm{Mg}$ concentration.

InGaN is used as an active material in optical devices, however its physical properties and influence on device performance have not been fully understood. InGaN is known to have 
compositional inhomogeneity and efforts have been made to suppress the inhomogeneity in order to improve device performance [8]. This inhomogeneity in InGaN quantum wells is thought to lead to a broadening in the spontaneous photoluminescence spectra. The gain and electrically pumped luminescence spectra from InGaN active layers are broader compared to other semiconductor materials used in optical devices [9]. In this paper, we use TEM to describe the microstructure of the InGaN layers together with x-ray studies, using high resolution x-ray diffraction to show that for a wide range of In concentration two sub-layers (strained and relaxed) are formed and the In concentration in the strained layer is much lower than the nominal value. Relaxed layers with planar defects show an In concentration very close to the nominal value. Two distinct lattice parameters can be observed by $\mathrm{x}$-ray diffraction only when asymmetrical reflections are used. Symmetrical (0002) reflections do not show this property.

\section{B. Experimental}

Different types of $\mathrm{GaN}$ crystals doped by $\mathrm{Mg}$ have been studied using plan-view and cross-sectioned samples. Bulk crystals were grown by the High Nitrogen Pressure Method [10] from a solution of liquid gallium containing $0.1-0.5$ at. $\% \mathrm{Mg}$ [11]. Two types of samples were grown using MOCVD where Mg was added ether continuously during the growth or where $\mathrm{Mg}$ was added by the delta doping method. The details of this growth has been described earlier [6-7]. Following the superlattice growth, the temperature was lowered to $850^{\circ} \mathrm{C}$, the ambient was switched to nitrogen only, and a 10 minute in-situ anneal was performed to dissociate the $\mathrm{Mg}-\mathrm{H}$ complexes and activate the $\mathrm{Mg}$ atoms [12]. The same growth temperature and post annealing was applied to the crystals for which $\mathrm{Mg}$ was added continuously during growth.

$\mathrm{In}_{\mathrm{X}} \mathrm{Ga}{ }_{1-\mathrm{X}} \mathrm{N}$ layers were grown on (0001)-oriented sapphire substrates using a specially designed atomic layer epitaxy (ALE)/metatorganic chemical vapor deposition (MOCVD) reactor described earlier [13]. An ALE buffer layer was first grown on the substrate followed by the deposition of a $250 \mathrm{~nm}$ thick $\mathrm{GaN}$ layer. The InGaN layers were finally grown on top of this GaN layer. Three different samples corresponding to In mole fractions of 28, 40 and $45 \%$ respectively were subsequently grown by controlling the deposition temperature and the hydrogen flow rate [13]. The samples with nominal $\mathrm{InN}$ concentration of $28 \%$ and $40 \%$ respectively were grown using a $\mathrm{GaN}$ buffer layer. The third one with $45 \% \mathrm{InN}$ was grown with an AlN buffer layer.

All GaN:Mg and the InGaN crystals have been studied using TEM on plan-view and cross-section samples, transparent for electrons, prepared by standard methods. The InGaN crystals were also studied using double-crystal and triple-axis x-ray diffractometry. X-ray diffraction measurements were performed using the Philips High Resolution diffractometer in double (DCD optics) and triple configuration (TAD optics). The primary beam was monochromatized by four 022 reflections from Ge channelcut crystals. For TEM studies two different microscopes were used: Topcon 002B and JEOL ARM with the acceleration voltage 200 and $800 \mathrm{kV}$, respectively.

\section{Results}

C.1. Bulk Crystals

A strong dependence of structure on crystal growth polarity was observed in bulk platelete crystals. Generally for the crystal sides growing in the direction from $\mathrm{N}$ to $\mathrm{Ga}(\mathrm{N}$ polarity) Mgrich planar defects were observed and for growth in the opposite polar direction pyramidal defects also rich in $\mathrm{Mg}$ with the base on c-plane and six planes inclined $45^{\circ}$ to the 
basal plane. Some rectangular defects delineated by eplanes are also observed for the growth with Ga polarity.

On the N-polar sides of the platelete some parts of the crystals can be perfect without any defects while in other samples planar defects can be formed despite the fact that $\mathrm{Mg}$ concentrations determined by SIMS remain practically unchanged. These defects can be perfectly ordered. They can form a perfect array of planar defects separated from each other by 20 unit cells of GaN [5-7]. However, in some crystals this perfect ordering can be interrupted by larger areas of hexagonal $\mathrm{GaN}$ as is shown on (Fig. 1a) or this ordering can be intercepted by another type of planar defect ( Fig. 1b). Perfect ordering leads to superdiffraction spots dividing the (0001) lattice distance into 20 equal parts. High resolution images from these show a $1 / 3[1 \overline{1} 00]+\mathrm{c} / 2$ displacement [5-7] which could characterize these defects as stacking faults.
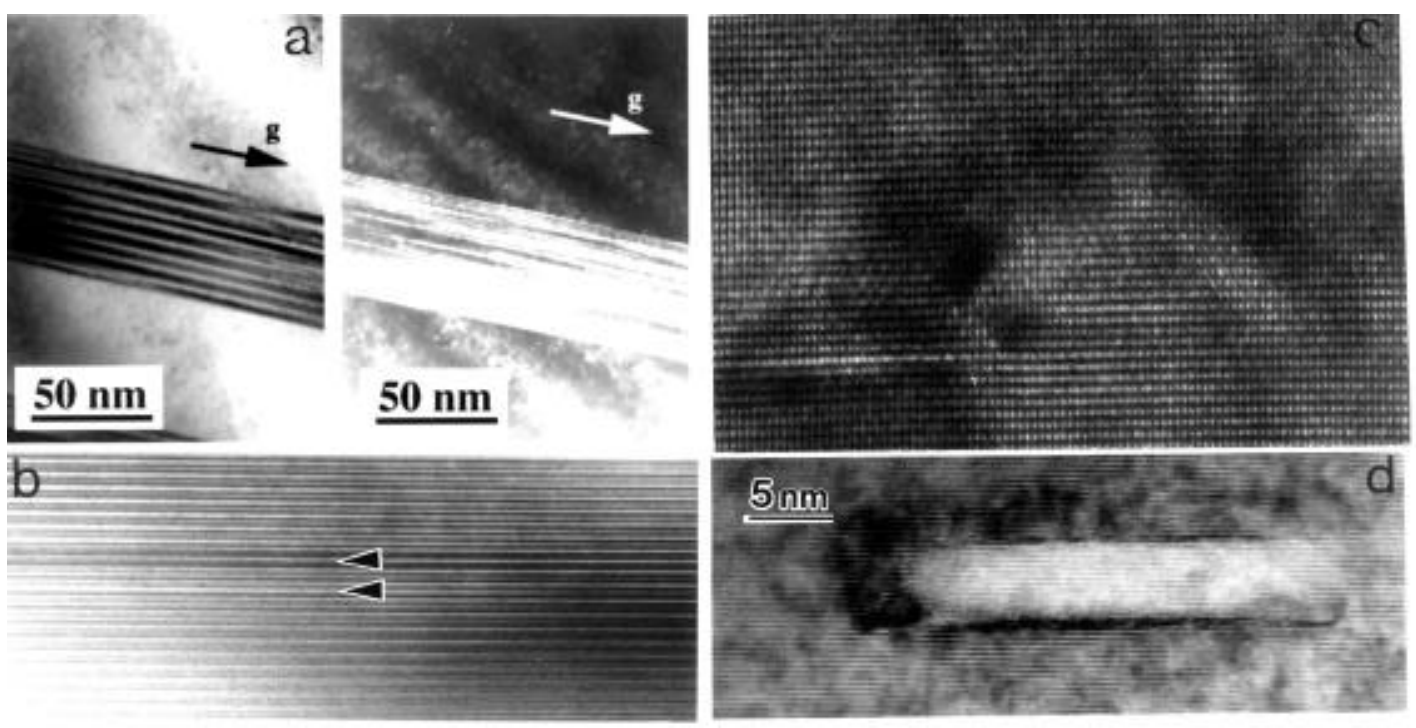

Fig. 1. TEM cross-section micrographs showing defects formed in GaN:Mg for the growth with $\mathrm{N}$-polarity (a and b) and with Ga-polarity (c and d)

However, split (0001) and (0003) reflections and reverse contrast for (0001) and $(000 \overline{1})$ reflections used for a multi-beam dark field image obtained from the [11 $\overline{2} 0]$ zone axis [5] suggest that these defects might be inversion domains with a very narrow width along the c-axis. The pairs of inversion boundaries are separated by not more than 3 unit cells. These defects extend through the whole width of the crystal, therefore, it is impossible to see partial dislocations which would help to characterize them. One of the domain boundaries called the flat interface (FI) shown in Figs .(2a,b) always remains on the same plane. The upper boundary, called the corrugated interface, changes its position so that the domain thickness is changing from $3 / 2 \mathrm{c}$ to $5.2 \mathrm{c}$ as is shown on high resolution images taken from the same defects.

The opposite side of the bulk crystals (grown with Ga-polarity) had a completely different defect structure as shown in Figs. (1c and d). They appear in [11 $\overline{2} 0]$ cross-section TEM micrographs as triangular features with a base on the (0001) eplane and six side facets. All these triangles were oriented in a direction with the base closest and parallel to the sample surface with Ga-polarity, e.g. from the triangle tip to the base a long bond direction along the c-axis is from $\mathrm{Ga}$ to $\mathrm{N}$. 

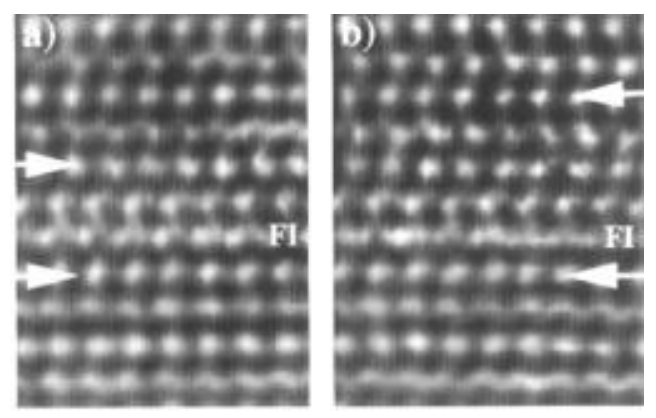

Fig. 2. Two areas of the same planar defect showing the change in defect thickness (indicated by arrows) along the eaxis. The flat interface (FI) remains always on the same eplane while the upper one changes.

No satellite spots were observed on this side of the crystal. Studies in plan-view configuration confirm that these defects are pyramids and that they are empty inside [6-7]. Convergent beam electron diffraction studies confirm $\mathrm{Mg}$ segregation on the defect walls. The dimension of the largest defects varies. The longest base length of these defects are in the range $100 \mathrm{~nm}$ and the smallest about 3-5 nm. The density of these defects is in the range $2.5 \times 10^{9} \mathrm{~cm}^{-2}$. A second type of hollow defect (empty inside) was also observed in crosssection samples, a rectangular defect (Fig. 1d) delineated by planar defects formed basal planes [6-7].

\section{C.2. Layers grown by MOCVD}

Since the majority of layers applied in devices are grown by MOCVD it was also interesting to observe the structure of p-doped layers to learn why p-doping is so difficult [12]. Application of $\mathrm{GaN}$ in devices would have developed much earlier, if the p-doping would be more successful [14]. Two types of crystals have been studied: one where $\mathrm{Mg}$ was added as delta doping and the second where $\mathrm{Mg}$ was added continuously.

TEM studies on cross-section Mg-delta doped samples also show both types of defects observed for the two opposite growth polarities in bulk GaN:Mg, e.g. planar defects (polytypoids) observed earlier for $\mathrm{N}$ growth polarity and pyramidal and rectangular pinholes observed in the bulk GaN:Mg for growth with Ga polarity [5-7]. In two different crystals with Mg-delta doping planar defects were formed followed by a high density of pyramidal defects. In one crystal a layer about $100 \mathrm{~nm}$ thick with planar defects, like those observed in bulk crystals grown with N-polarity was observed at about $150 \mathrm{~nm}$ distance from the buffer layer [6]. Further growth resulted in a random distribution of pyramidal defects with a density ( $10^{10} \mathrm{~cm}^{-2}$ ). In the second crystal planar defects were also formed at a $300 \mathrm{~nm}$ distance from the buffer layer (Fig. 3a), but they did not formed an ordered structure. They were followed by a high density of pyramidal defects arranged laterally. This layer-like arrangement of these pyramidal defects was observed only for a distance of about $400 \mathrm{~nm}$ from the buffer layer. Further growth resulted in the random distribution of these defects. SIMS analysis indicated that $\mathrm{Mg}$ concentration was steadily increasing and in the area with planar defects it reached the level of $4 \times 10^{19} \mathrm{~cm}^{-3}$. In the area where the hollow defects (rectangular and triangular) were formed $\mathrm{Mg}$ concentration dropped to $2 \times 10^{19}$ atoms $/ \mathrm{cm}^{3}$ and stayed almost constant with a slow increase to reach at the sample surface a concentration of $4 \times 10^{19} \mathrm{~cm}^{-3}$ [7]. 


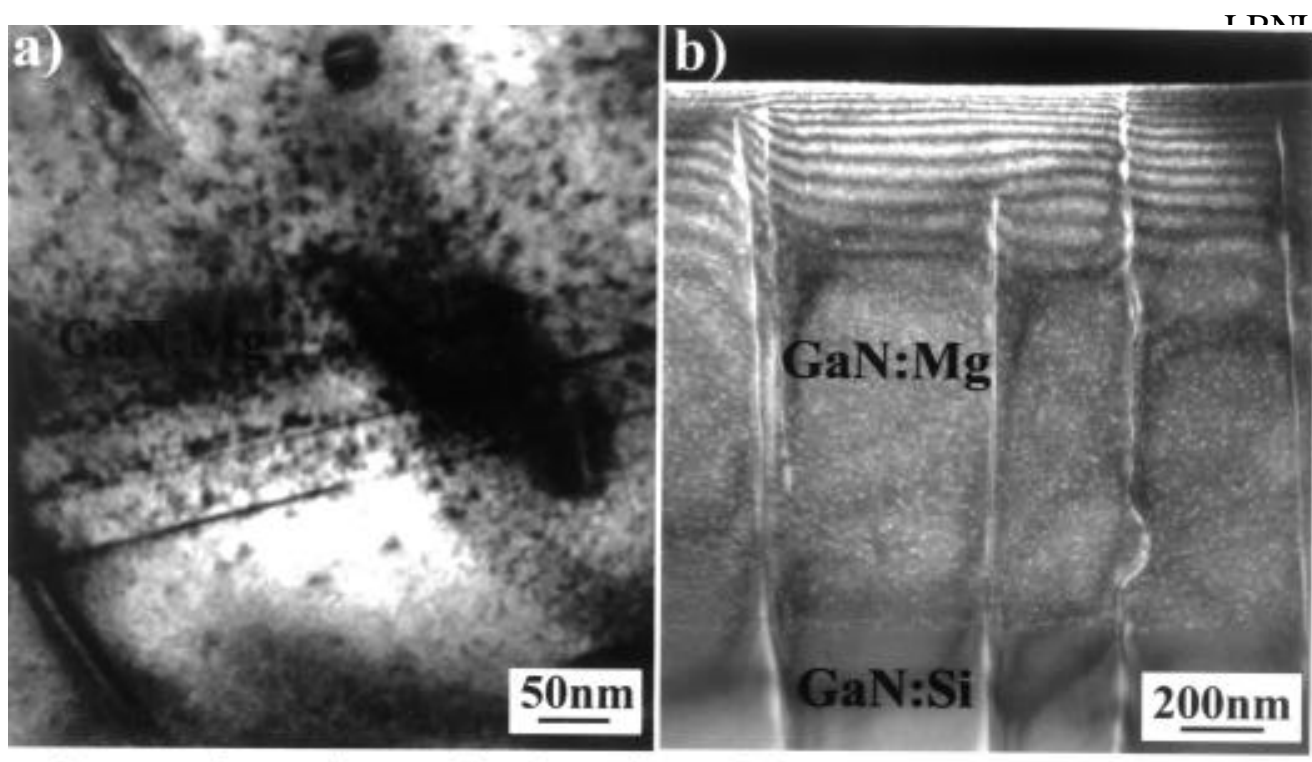

Fig. 3. Cross-section micrographs from the MOCVD-grown samples; (a) with $\mathrm{Mg}$ delta doping where planar a three-dimensional defects are formed; (b) with continuous $\mathrm{Mg}$ doping where high density of three-dimensional defects are observed.

In the samples where $\mathrm{Mg}$ was added ontinuously (with comparable $\mathrm{Mg}$ concentration to that in the previous samples) no planar defects were observed but a high density of pyramidal defects was present (Fig. 3b). These pyramids are also empty inside but their size was much smaller ( $8 \mathrm{~nm}$ in the base). At the base of these defects stacking faults were not formed as in the larger pyramids observed in the bulk samples. However, the expansion of the lattice parameter of about $15 \%$ was observed in two basal parallel layers. These expansion agrees well with what would be expected if $\mathrm{Ga}$ atoms were substituted by $\mathrm{Mg}$.

\section{Discussion of $\mathrm{Mg}$ results}

TEM studies of bulk crystals grown under high pressure and high temperature doped with $\mathrm{Mg}$ and those grown by MOCVD show formation of structural defects for a wide range of $\mathrm{Mg}$ concentration. This would explain (at least to some extend) why is so difficult to obtain high hole concentration despite nominal higher $\mathrm{Mg}$ concentration in the crystal. It appears also that generally two types of defects are formed in GaN:Mg e.g. planar defects and threedimensional defects: pyramids empty inside (or rectangular also empty inside). Formation of these different defects is influenced by crystal growth polarity. This would be consistent with theoretical calculations by Bungaro at al [15] showing that the $\mathrm{Mg}$ arrangement in the $\mathrm{GaN}$ subsurface layer is dependent on crystal growth polarity and on the environment in which the crystal is growing (N-rich conditions vs. Ga-rich conditions). For a Ga-polarity surface in a $\mathrm{N}$-rich environment $\mathrm{Mg}$ would substitute $\mathrm{Ga}$ sites $\left(\mathrm{Mg}_{\mathrm{Ga}}\right)$ and a $\mathrm{Ga}$ atom would be shifted to the surface. It is also expected that growth with Ga-polarity would be more favorable than growth with $\mathrm{N}$-polarity. This is consistent with our studies which show much faster growth on the surface with Ga polarity.

Ordering of planar defects observed in GaN:Mg formed for growth with N-polarity appears to be similar to the polytypoids formed in AIN rich in oxygen. However, defects formed in the MOCVD GaN samples are practically the same as those in the bulk samples despite the fact that oxygen concentration is three orders of magnitude lower $\left(5 \times 10^{16} \mathrm{~cm}^{-3}\right.$ in comparison to $5 \times 10^{19} \mathrm{~cm}^{-3}$ in the bulk samples). Therefore, the polytypoids which are formed in our GaN:Mg samples must be caused by the $\mathrm{Mg}$ presence, not by oxygen as proven by EDX studies [5]. A pair of inversion boundaries appear to be formed-a flat boundary which 
persist on the same c-plane through the whole crystal and an upper corrugated boundary which is changing its $\mathrm{c}$ plane from place to place leading to different domain thickness along the c-axis. However, the thickness of such domains does not exceed more than about 3 unit cells along c-axis. No inversion in the GaN between these planar defects is observed.

\section{E. $\operatorname{In}_{\mathbf{x}} \mathrm{Ga1-x} \mathrm{N}$ layers grown with different In composition}

Since In is used in the active parts of devices and influences wavelength of the luminescence spectra TEM and x-ray studies were applied to samples with increasing In concentrations in the nominal range 28 to $45 \%$. TEM studies of cross-section samples show that even for the lower In concentration the surface of the layer is not smooth and characteristic V-shaped defects delineated by $(10 \overline{1} 1)$ planes are observed (Fig. 4a).

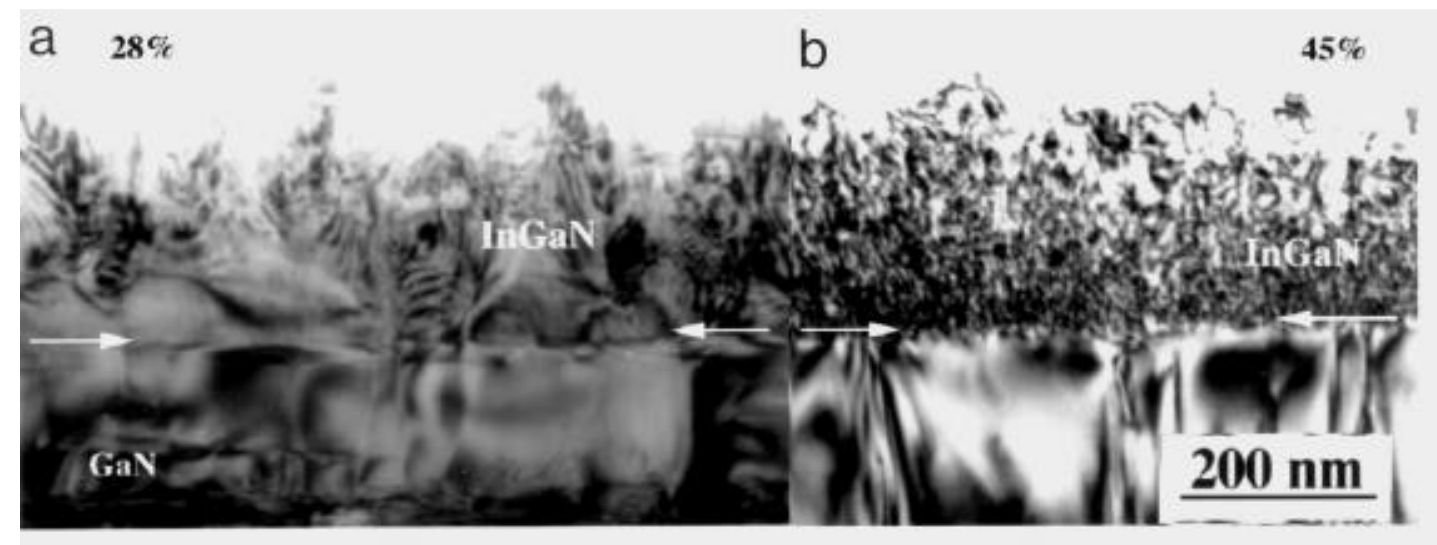

Fig. 4. TEM cross-section micrographs (a) for sample with nominal $28 \%$ In with two sublayers, one almost perfect close to the $\mathrm{GaN}$ and the second with planar defects; (b) for sample with nominal $45 \%$ In with planar defects in the whole layer.

The surface becomes rougher with an increased In composition. For the sample with the $28 \%$ In it is observed that the InGaN layer is divided into two sub-layers that exhibit different contrast (Fig. 4a). The first sub-layer (40 nm thick) grown directly on the GaN has good quality with only those defects which propagate from the underlying GaN layer. On top, the second sub-layer of about $200 \mathrm{~nm}$ thick is very defective. The defects are stacking faults formed on c-planes which lead to sample relaxation. Similarly two sub-layers were observed in the sample grown with $40 \%$ In, but the first sub-layer was much thinner. The third sample with 45\% In grown with an AlN buffer layer (instead of GaN as was applied to the first two samples) was relaxed and only one $150 \mathrm{~nm}$ thick layer with a high density of stacking faults was observed in TEM cross-section samples (Fig. 4b). Both, low energy stacking faults with one zinc-blende unit and high energy stacking faults with three zinc-blende units were observed and the distance between these defects was in the range of $3 \mathrm{~nm}$.

X-ray rocking curves and reciprocal mapping were applied to determine In concentration and samples quality. Lattice parameters were measured based on Vegard's law. The reference lattice parameters of $\mathrm{GaN}$ and $\mathrm{InN}$ were taken from [16] $\mathrm{a}_{\mathrm{GaN}}=3.182(9) \AA$, $\mathrm{c}_{\mathrm{GaN}}=5.1850(5) \AA, \mathrm{a} \mathrm{InN}=3.5838 \AA \mathrm{C}_{\mathrm{InN}}=5.703 \AA$ In order to determine the relaxed values of the lattice parameters ( $\mathrm{a}$ and $\mathrm{c}$ ), 0002, 0006, $\overline{2} 1 \overline{1} 4$ and $01 \overline{1} 5$ reflections have been used. More detailed information about strain status of the layers was obtained form reciprocal space mapping. From the 0002 symmetrical reciprocal space maps (not shown for the lack of space) it can be concluded that lattice planes of the layers are not tilted in respect to those of the $\mathrm{GaN}$ 
layer. Asymmetrical space maps for $\overline{2} 1 \overline{1} 4$ reflection presented in Fig. 5 illustrate the strain status of the samples. It is clearly shown that for the samples I and II with 28 and $40 \%$ In , respectively, two different lattice parameters can be assigned to the $\mathrm{InGaN}$ layer. This suggested that these In-rich layers consist of two sub-layers with different indium concentrations and different strain value with respect to $\mathrm{GaN}$, consistent with the TEM observation. These studies show that much smaller In concentration is detected in the strained sub-layers in comparison to the relaxed sub-layers. The strained sub-layers contained $17 \%$ In (sample I) and 23\% In (sample II), much smaller than the nominal values $28 \%$ and $40 \%$.
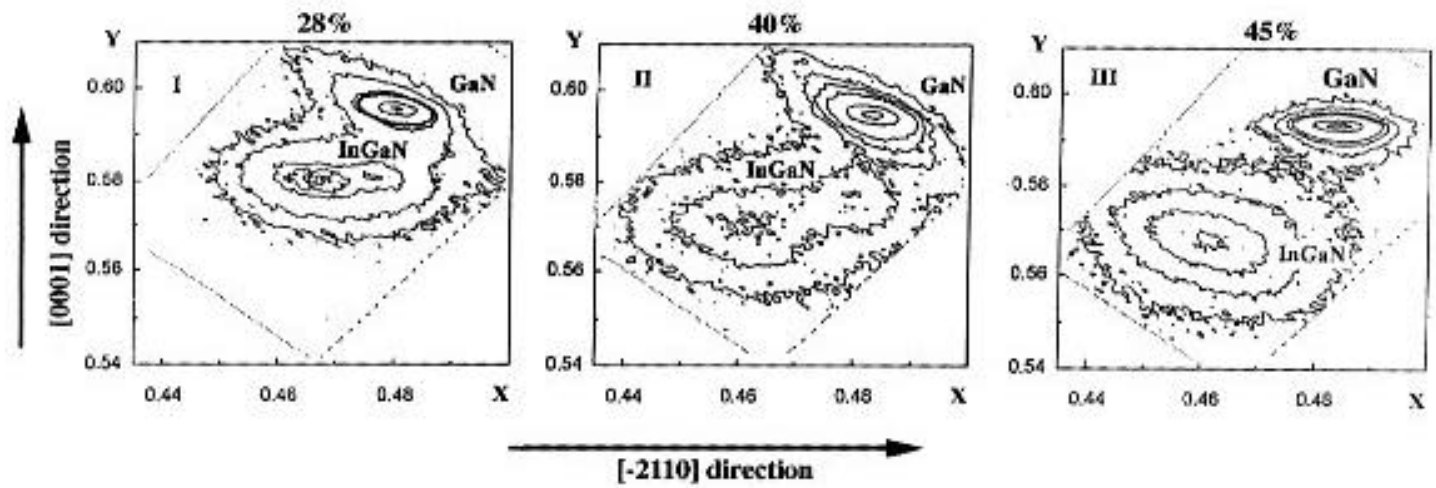

Fig. 5. Reciprocal space maps for asymmetrical $\overline{2} 1 \overline{1} 4$ reflections.

Indium concentrations in the relaxed sub-layers for samples I and II were $27.2 \%$ and $37.4 \%$, respectively, close to the nominal values. The relaxed sample III with a high density of stacking faults contains $43.8 \% \mathrm{In}$, close to the nominal value of $45 \%$. A full description of this study will be published separately [17]. This different In concentration and different lattice parameters can be observed only for asymmetrical reflections, not for the commonly used symmetrical (0002) reflection, since the 'a' lattice parameter is changing more than the 'c' lattice parameter. This study suggests use of asymmetrical reflections for sample characterization, especially when values of strain are compared to data obtained by photo luminescence when red or blue shift is observed.

\section{F. Summary}

TEM studies of Mg-doped GaN crystals show formation of structural defects for a wide range of $\mathrm{Mg}$ concentration. The type of defects formed is strongly influenced by crystal growth polarity. Similar defects are formed for bulk crystals grown under high hydrostatic pressure and for those grown heteroepitaxially on sapphire using MOCVD. In some bulk crystals for the plate side grown with $\mathrm{N}$-polarity a "microsuperlattice" which leads to satellite diffraction spots dividing the (0001) reciprocal lattice distance into 20 parts was observed. Three-dimensional defects, pyramidal and rectangular, were observed for growth with Ga polarity also due to $\mathrm{Mg}$ segregation. This indicates that $\mathrm{Mg}$ segregated on particular planes can prevent further growth. These defects were observed in the majority of bulk GaN samples and in all samples grown by MOCVD. When these pyramids are small a $15 \%$ lattice expansion can be detected in the bases of the pyramids consistent with the substitution of $\mathrm{Ga}$ by $\mathrm{Mg}$ atoms. Further growth of these pyramids leads to the formation of a stacking fault near the base of this defect.

TEM studies together with $x$-ray studies showed that for a wide range of In concentration in InGaN layers are divided into two sub-layers. The layer adjacent to the underlying $\mathrm{GaN}$ is strained and has a much lower In concentration compared to the nominal 
composition. A relaxed sub-layer formed on top of the strained layer with a high density of stacking faults has an In concentration very close to the nominal value. Determination of the lattice parameters in these layers was much easier to observe using asymmetrical x-ray reflections. This is useful especially when results are compared with PL data.

\section{Acknowledgment}

This work was supported by the Director, Office of Basic Science, Materials Science Division, U.S. Department of Energy, under the Contract No. DE-AC03-76SF00098. The use of the facility at the National Center for Electron Microscopy at E.O. Lawrence Berkeley National Laboratory is greatly appreciated. This work at UT-Austin was partially supported by the Office of Naval Research under N00014-95-1-1302 (monitored by Dr. J. C. Zolper) and the National Science Foundation under Grant CHE-89-20120.

\section{References:}

1. H. Amano, M. Kito, K. Hiramatsu, and I. Akasaki, Inst. Phys. Conf. Ser. 106, (1989) 725

2. S. Nakamura, N. Iwasa, M. Senoh, and T. Mukai, Jpn. J. Appl. Phys. 31, (1992) 1258.

3. S. Nakamura, M. Senoh, and T. Mukai, Jpn. J. Appl. Phys. 31, (1991) L1708.

4. S. Nakamura, Paper Plenary 1, presented at the 24th International Symposium on Compoud Semiconductors, San Diego CA, 811 September 1997.

5. Z. LilientatWeber, M. Benamara, J. Washburn, I. Grzegory, and S. Porowski, Phys. Rev. Lett. 83, (1999) 2370.

6. Z. LilientatWeber, M. Benamara, W. Swider, J. Washburn, I. Grzegory, S. Porowski, D.J.H. Lambert, C.J. Eiting, and R.D. Dupuis, Appl. Phys. Lett. 75, (1999) 4159.

7. Z. LilientatWeber, M. Benamara, W. Swider, J. Washburn, I. Grzegory, S. Porowski, D.J.H. Lambert, C.J. Eiting, and R.D. Dupuis, Physica B 273-274, (1999) 124.

8. S. Chichibu, K. Wada and S. Nakamura Appl. Phys. Lett. 71, (1997) 2346.

9. H. Sakai, T. Takeuchi, S. Sota, M. Katsuragawa, M. Komori, H. Amano, and I. Akasaki, Proc. Second Int. Conf. Nitride Semicond, 1997, Tokushima, S.5.

10. I. Grzegory , J. Jun, M. Bockowski, S. Krukowski, M. Wroblewski, B. Lucznik, and S. Porowski, J. Phys. Chem. Solids 56, (1995) 639.

11. S. Porowski, M. Bockowski, B .Lucznik, I. Grzegory, M. Wroblewski, H. Teisseyre, M. Leszczynski, E. Litwin-Staszewska, T .Suski, P. Trautman, K. Pakula and J.M. Baranowski, Acta Physica Polonica A 92, (1997) 958.

12. C.J. Eiting, P.A. Grudowski, J.S. Park, D.J.H. Lambert, B.S. Shelton, and R.D. Dupuis, J. Electrochem. Soc. 144, (1997) L219.

13. N. A. El-Masry, E. L. Piner, S. X. Liu and S. M. Bedair, Appl. Phys. Lett. 72, (1998) 40.

14. J. I. Pankove, E.A Miller, D. Richman, and J .E . Berkeyheiser, J.Lumin., 4, (1971) 63.

15. C. Bungaro, K. Rapcewicz, and J. Bernholc, Phys. Rev. B59, (1999) 9771.

16. "Properties, processing and applications of $\mathrm{GaN}$ and related semiconductors", edited by J. H. Edgar et al., Datareviews Series No 23, (London 1999).

17. Z. LilientatWeber, M. Benamara, J. Washburn, J.Z. Domagala, J. Bak-Misiuk, E.L. Piner, J.C. Roberts and S. M. Bedair, J. Electr. Mat. subm. 